\title{
ASYMPTOTIC EXPANSION OF THE HEAT KERNEL FOR A CLASS OF HYPOELLIPTIC OPERATORS
}

\author{
ALEXANDER LOPATNIKOV \\ Space Research Institute, Russian Academy of Sciences \\ Profsoyuznaya 84/32, Moscow, 117810 Russia
}

Introduction. Let $M$ be a smooth compact manifold without boundary, let $d x$ be a fixed positive smooth density on $M$, and let $X_{1}, \ldots, X_{l}$ be smooth real vector fields on $M$, i.e. in a local coordinate system, $X_{j}=\sum_{i=1}^{n} a_{j}^{i} \partial_{x_{i}}$. We will consider operators $A$ of the form ( $m$ is even)

$$
(-1)^{m / 2} \sum_{j=1}^{l} X_{j}^{m}+\sum_{|\alpha|<m} a_{\alpha}(x) X^{\alpha}
$$

where $\alpha=\left(\alpha_{1}, \ldots, \alpha_{k}\right), X^{\alpha}=X_{\alpha_{1}} \ldots X_{\alpha_{k}},|\alpha|=k$ and the $a_{\alpha}$ are smooth functions. The well-known example is the sum of the squares of vector fields,

$$
-\sum_{j=1}^{l} X_{j}^{2}+X_{0}+c(x) .
$$

A result of Hörmander $[7,14]$ states that this operator is hypoelliptic if the vector fields $X_{1}, \ldots, X_{l}$ and all their commutators $\left[X_{i_{1}},\left[X_{i_{2}} \ldots\left[X_{i_{s-1}}, X_{i_{s}}\right] \ldots\right], s \leq r\right.$, up to length $r$ span the tangent space to $M$ at each point. We recall that an operator $A$ is said to be hypoelliptic on $M$ if for any open set $U \subset M$ and distributions $u, f$ on $U$ satisfying $A u=f, f \in C^{\infty}(U)$ implies $u \in C^{\infty}(U)$. In [17] for the operator (2) it was shown (with $m=2$ ) that

$$
\|u\|_{m / r} \leq C\left(\|A u\|_{0}+\|u\|_{0}\right)
$$

for all $u \in C^{\infty}(M)$, where $\|\cdot\|_{s}$ denotes the norm in the usual Sobolev space $H_{s}(M)$. For the operator (1) this estimate and hypoellipticity were proved in $[6$, $16]$.

We assume that the operator (1) is formally selfadjoint and positive, that is, $(A u, v)=(u, A v)$ and $(A u, u) \geq 0$ for all $u, v \in C^{\infty}(M)$. It is easy to show that 
under our assumption $A$ is an unbounded selfadjoint operator on the domain $D_{A}=\left\{u \in H_{m / r}(M): A u \in L_{2}(M)\right\}$ and has discrete spectrum $\lambda_{j} \rightarrow \infty$. Let $U(x, y, t)$ be the kernel of the operator $\exp (-t A)$,

$$
U(x, y, t)=\sum_{j=1}^{\infty} e^{-\lambda_{j} t} \varphi_{j}(x) \varphi_{j}(y)
$$

Here $\varphi_{j}(x)$ is a complete orthonormal set of eigenfunctions of $A$ with eigenvalues $\left\{\lambda_{j}\right\} . U(x, y, t)$ is a fundamental solution for the operator $L=\partial_{t}+A$ and so it is called the heat kernel for $L$.

We denote by $V_{k}(x)$ the subspace of $T_{x}(M)$ spanned by $X_{1}, \ldots, X_{l}$ and all their commutators of length $\leq k$ and let $\nu_{k}(x)=\operatorname{dim} V_{k}(x)\left(\nu_{0}=0\right)$. We say that Hörmander's condition (of order $r$ ) holds if

$$
\nu_{r}(x)=\operatorname{dim} M=n \quad \text { for all } x \in M .
$$

We will also use the condition introduced by Métivier [13]:

$$
\nu_{k}(x)=\nu_{k}=\text { const }, \quad 1 \leq k \leq r, \text { for all } x \in M .
$$

Our main results are the following.

THEOREM 1. If Hörmander's condition holds then the heat kernel for the operator (1) has the following asymptotic expansion as $t \rightarrow+0$ :

$$
U(x, x, t)=\sum_{j=-q(x)}^{\infty} c_{j}(x) t^{j / m}+\sum_{j=0}^{\infty} d_{j}(x) t^{j / m} \ln (t)
$$

where $q(x)=\sum_{k=1}^{r}\left(\nu_{k}(x)-\nu_{k-1}(x)\right) k$, and $c_{j}(x), d_{j}(x)$ are some functions on $M$.

We remark that in general this expansion is not uniform in $x \in M$ and the functions $c_{j}, d_{j}$ are not continuous.

TheOREM 2. In the Métivier case the asymptotics in Theorem 1 is uniform in $x \in M, c_{j}(x), d_{j}(x) \in C^{\infty}(M)$, and as $t \rightarrow+0$,

$$
\operatorname{tr} \exp (-t A)=\sum_{j=-q}^{\infty} c_{j} t^{j / m}+\sum_{j=0}^{\infty} d_{j} t^{j / m} \ln (t)
$$

where $q=\sum_{k=1}^{r}\left(\nu_{k}-\nu_{k-1}\right) k$.

It is also possible to find the leading coefficient $c_{-q(x)}(x)$ explicitly (see below). For elliptic operators this result is well known; in this case $q=\operatorname{dim} M$ and in addition all $d_{j}=0$. For the operator (2) our results were obtained independently by G. Ben Arous [2, 3], who used probabilistic methods. Formula (4) was also proved in [19] for $r=2$, and related results were obtained in $[1,18]$. D. Jerison and A. Sánchez-Calle $[8,9,15]$ estimated the kernel $U(x, y, t)$ in terms of the metric associated with the operator $A$. From the asymptotics of the heat kernel it is easy to find the first term of the asymptotics of the spectral function of $A$ [10-12] (for second order operators in the Métivier case this was done by a different method 
by G. Métivier [13]). To prove Theorems 1,2 we use the method developed in $[3$, $7,8]$.

1. Dilations and homogeneity. In this section we recall some definitions and propositions connected with homogeneous structures (see [4, 5, 15, 17] for details). Let $e_{1}, \ldots, e_{n}$ be a basis in $\mathbb{R}^{n}$ and let $0=\nu_{0}<\nu_{1}<\ldots<\nu_{r}$ be integers. We write $[j]=k$ if $\nu_{k-1}<j \leq \nu_{k}$. We define a group of linear automorphisms $\delta_{s}$ of $\mathbb{R}^{n}$ by

$$
\delta_{s}\left(e_{j}\right)=s^{[j]} e_{j}, \quad 1 \leq j \leq n .
$$

We also consider a homogeneous norm $\|\cdot\|$ with respect to $\delta_{s}$ such that

$$
\|u\|=0 \Leftrightarrow u=0, \quad\left\|\delta_{s}(u)\right\|=s\|u\| .
$$

For example we can take $\|u\|=\left(\sum_{j=1}^{n}\left|u_{j}\right|^{2 /[j]}\right)^{1 / 2}$. This norm satisfies the following inequalities:

$$
\|u+v\| \leq C(\|u\|+\|v\|), \quad C_{1}|u| \leq\|u\| \leq C_{2}|u| \quad \text { for }|u| \leq C,
$$

where $|\cdot|$ is the usual euclidean norm in $\mathbb{R}^{n}$. The number $q=\sum_{k=1}^{r}\left(\nu_{k}-\nu_{k-1}\right) k$ is called the homogeneous dimension of the space. It is easy to see that $\mathbb{R}^{n}=$ $\bigoplus_{k=1}^{r} V_{k}, V_{k}$ is spanned by the vectors $e_{j}$ for $[j]=k$, and $q=\sum_{k=1}^{r} k \operatorname{dim} V_{k}$.

A function $f$ is homogeneous of degree $\lambda$ if $f \circ \delta_{s}=s^{\lambda} f$ for all $s>0$. A distribution $v$ is homogeneous of degree $\lambda$ if $\left\langle v, \varphi \circ \delta_{s}\right\rangle=s^{Q-\lambda}\langle v, \varphi\rangle$. A function $k(u)$ is said to be a kernel of type $\lambda$ if it is smooth away from the origin and homogeneous of degree $-Q+\lambda$. A differential operator $T$ is homogeneous of degree $\lambda$ if $T\left(f \circ \delta_{s}\right)=s^{\lambda}(T f) \circ \delta_{s}$ for all $s>0$. For example, the function $u^{\alpha}=$ $u_{1}^{\alpha_{1}} \ldots u_{n}^{\alpha_{n}}$ is homogeneous of degree $[\alpha]=\sum_{j=1}^{n} \alpha_{j}[j]$, the operator $u^{\alpha} \partial / \partial u_{j}$ is homogeneous of degree $[j]-[\alpha]$. Let $U$ be a neighborhood of the origin in $\mathbb{R}^{n}$. We define the function space

$$
C_{m}^{\infty}(U)=\left\{f(u) \in C^{\infty}(U):|f(u)|=O\left(\|u\|^{m}\right), u \rightarrow 0\right\} .
$$

A differential operator $T=\sum_{|\alpha| \leq k} a_{\alpha}(u) \partial_{u}^{\alpha}$ from $C^{\infty}(U)$ to $C^{\infty}(U)$ is said to have degree at most $p$ at 0 whenever $T\left(C_{m}^{\infty}(U)\right) \subset C_{m-p}^{\infty}(U)$ for all $m \in \mathbb{N}$. For such an operator it is possible to define an operator $\widehat{T}$,

$$
\widehat{T}=\sum_{|\alpha| \leq k} \sum_{[\beta] \leq[\alpha]-p}\left(\partial_{u}^{\beta} a_{\alpha}(0) u^{\beta} / \beta !\right) \partial_{u}^{\alpha},
$$

which is homogeneous of degree $p$. The operator $T-\widehat{T}$ has degree at most $p-1$ at 0 .

Let $\mathfrak{g}$ be a free nilpotent Lie algebra of step $r$ with $l$ generators, $\mathfrak{g}=\bigoplus_{k=1}^{r} \mathfrak{g}_{k}$, and $\left[\mathfrak{g}_{i}, \mathfrak{g}_{j}\right]=\mathfrak{g}_{i+j}$ if $i+j \leq r,\left[\mathfrak{g}_{i}, \mathfrak{g}_{j}\right]=0$ if $i+j>r$. Using the exponential mapping we can identify $\mathfrak{g}$ and the corresponding Lie group $G$; the group multiplication in $\mathfrak{g}$ will be given by the Campbell-Hausdorff formula

$$
u \cdot v=u+v+[u, v]+\ldots, \quad u, v \in \mathfrak{g} .
$$


Following [15] we now define a function class $F_{\lambda}: k \in F_{\lambda}$ if

(i) $k \in C^{\infty}(\mathfrak{g} \backslash 0), k(u)=0$ for $\|u\|>1$,

(ii) $|P k(u)| \leq C_{s}\left(1+\|u\|^{\lambda-Q-s}\right)$, for all left-invariant differential operators $P$ homogeneous of degree $s$.

We will also use another class $H F_{\lambda}$. A function $k$ is in $H F_{\lambda}$ if $k \in F_{\lambda}$ and

(i) if $\lambda<Q$ then $k(u)=\widehat{k}(u)+g(u)$, where $\widehat{k}(u)$ is a kernel of type $\lambda, g(u) \in$ $C^{\infty}(\mathfrak{g})$,

(ii) if $\lambda \geq Q$ then (i) holds for the function $P k$ for all left-invariant differential operators $P$ of degree $s, \lambda-s<Q$.

Lemma 1. 1) If $k$ is a kernel of type $\lambda, \varphi \in C_{0}^{\infty}(\mathfrak{g})$ and $\varphi(u)=1$ for $\|u\| \leq 1 / 2$, then $\varphi k \in H F_{\lambda}$ and $P \varphi k \in H F_{\lambda-s}$ for $P$ homogeneous of degree $s$.

2) If $k \in H F_{\alpha}, h$ is a kernel of type $\beta, 0<\beta<Q, \alpha>0$ and $\varphi \in C_{0}^{\infty}(\mathfrak{g})$ with $\varphi(u)=1$ for $\|u\| \leq 1 / 2$ then $\varphi(k * h) \in H F_{\alpha+\beta}$.

Proof. We have $\varphi k=\widehat{k}+g$ with $\widehat{k}=k$ and $g=(1-\varphi) k(u) \in C^{\infty}(\mathfrak{g})$ since $k(u)$ is a kernel of type $\lambda$, so $\varphi k \in H F_{\lambda}$. For $P \varphi k$ we observe that since $\varphi=1$ in a neighborhood of the origin, $P \varphi k-\varphi P k \in C_{0}^{\infty}$; since $P k$ is a kernel of type $\lambda-s$, we have $P \varphi k \in H F_{\lambda}$ and 1 ) is proved.

For 2) we observe that if $\alpha+\beta<Q$ then by definition $k(u)=\widehat{k}(u)+g(u)$, so $k * h(u)=\int k(v) h\left(v^{-1} u\right) d v=\int \widehat{k}(v) h\left(v^{-1} u\right) d v+\int g(v) h\left(v^{-1} u\right) d v=I_{1}+I_{2}$. $I_{1}$ is a kernel of type $\alpha+\beta$ by the result of Folland [4], $I_{2}(u)$ is a smooth function, and using the same arguments as in Lemma 3 of [15] one can see that $g=I_{2}$ satisfies the required estimate. In the case $\alpha+\beta \geq Q$ we have $P(k * h)=(P k) * h$, $P k$ is a kernel of type $\lambda-s$, and $\lambda-s+\beta<Q$, so as was shown before $\varphi P(k * h) \in$ $H F_{\alpha+\beta-s}$ and by definition $k * h \in H F_{\alpha+\beta}$.

We say that a function $k$ is in $S F_{\lambda}$ if for any $s \in \mathbb{N}$ with $s>\lambda$,

$$
k(u)=\sum_{j=0}^{s} k_{j}(u)+q_{s}(u)
$$

where $k_{j} \in H F_{\lambda+j}$ and $q_{s} \in F_{s}(\mathfrak{g})$.

2. Lifting of vector fields. Let $L(M)$ be the Lie algebra of smooth real vector fields on $M$. There exists a partial homomorphism $\mu: \mathfrak{g} \rightarrow L(M)$, that is, $\mu$ is linear and for all $a \in \mathfrak{g}_{i}, b \in \mathfrak{g}_{j}$ we have $\mu([a, b])=[\mu(a), \mu(b)]$ if $i+j \leq r$. Write $\mu_{x}(a)=\left.\mu(a)\right|_{x}, x \in M$.

We now define

$$
H_{k}(x)=\left\{a \in \mathfrak{g}_{k}: \mu_{x}(a) \in V_{k-1}(x)\right\}, \quad 1 \leq k \leq r, \quad H(x)=\bigoplus_{k=1}^{r} H_{k}(x) .
$$

We select $S_{k}(x)$ such that $\mathfrak{g}_{k}=H_{k}(x) \oplus S_{k}(x)$, and set $S(x)=\bigoplus_{k=1}^{r} S_{k}(x)$. As was shown in [5], $H(x)$ is a subalgebra in $\mathfrak{g}, \operatorname{dim} S_{k}(x)=\nu_{k}(x)-\nu_{k-1}(x)$ and 
$\operatorname{dim} S(x)=\operatorname{dim} M$. Obviously $q(x)$ is the homogeneous dimension of $S(x)$, and $q(x)+\beta(x)=Q$, where $\beta(x)$ is the homogeneous dimension of $H(x)$.

We now change the local coordinate system in a neighborhood of $x \in M$ so that in the new coordinates the vector fields $X_{1}, \ldots, X_{l}$ have degree at most one. It is easy to see that $S(x)=\mathfrak{g} / H(x)$; let $\gamma$ be a projection from $\mathfrak{g}$ to $S(x)$. The essential result in this situation is

Theorem 3 (Helffer-Nourrigat [5]). For any $x \in M$ there exists a diffeomorphism $\Theta_{x}: U \rightarrow \omega$, where $U$ is a neighborhood of 0 in $S(x)$ and $\omega$ is a neighborhood of $x$ in $M$, so that if $\mu(a)=X$ then

1) $\left(\widehat{\Theta_{x}^{-1}}\right)_{*} X=\widehat{X}, \quad \widehat{X} f(u)=\left.\frac{d}{d t}\right|_{t=0} f(\gamma(u \cdot t a))$;

2) $\left(\Theta_{x}(0)\right)_{*}(0)=\left.\mu_{x}\right|_{S(x)}$.

In the Métivier case $\Theta_{x}$ is smooth in $x \in M$.

We introduce coordinates $(u, v)$ in $\mathfrak{g}$ so that $u \in S(x), v \in H(x)$. If $\mu(a)=$ $X_{i}(1 \leq i \leq l)$ we define a left-invariant vector field $Y_{i}$ on $\mathfrak{g}$ by $Y_{i} f(u, v)=$ $\left.(d / d t)\right|_{t=0} f((u, v) \cdot t a)$. Consequently,

$$
Y_{i}(f \cdot \gamma)=\left.\frac{d}{d t}\right|_{t=0} f(\gamma((u, v)) \circ t a)=\left(\widehat{X}_{i} f\right) \circ \gamma .
$$

Let $R_{i}=X_{i}-\widehat{X}_{i}, 1 \leq i \leq l$. By Theorem 3 the vector fields $R_{i}$ have degree at most 0 at 0 . If we now define $\widetilde{X}_{i}=Y_{i}+R_{i}$ then we obtain

LEMma 2. $\widetilde{X}_{i}(f \circ \gamma)=\left(X_{i} f\right) \circ \gamma$ for $1 \leq i \leq l$.

3. Construction of the fundamental solution. We will consider two differential operators connected with $L$ :

$$
\widetilde{L}=(-1)^{m / 2} \sum_{j=1}^{l} \widetilde{X}_{j}^{m}+\sum_{|\alpha|<m} a_{\alpha}(x) \widetilde{X}^{\alpha}+\frac{\partial}{\partial t}, \quad \widehat{L}=(-1)^{m / 2} \sum_{j=1}^{l} Y_{j}^{m}+\frac{\partial}{\partial t} .
$$

LEMma 3. The operator $\widehat{L}$ is hypoelliptic.

P r o o f. For $m=2$ this follows directly from Hörmander's theorem. In the case $m>2$ it can be shown by using a criterion of hypoellipticity by Helffer-Nourrigat [6] (see also [12]).

On $\mathfrak{g}^{\prime}=\mathfrak{g} \times \mathbb{R}^{1}$ we define dilations by $\delta_{s}(\xi, t)=\left(\delta_{s}(\xi), s^{m} t\right), \xi \in \mathfrak{g}^{\prime}, t \in \mathbb{R}^{1}$. Then $Q^{\prime}=Q+m$ is the homogeneous dimension of $\mathfrak{g}^{\prime}$. For $\mathfrak{g}^{\prime}$ we can define the spaces $F_{\lambda}, H F_{\lambda}, S F_{\lambda}$ as in the previous section. It is clear that Lemma 1 is true in this situation. The operator $\widehat{L}$ is homogeneous on $\mathfrak{g}^{\prime}$ of degree $m$. By a result of G. B. Folland [4] we can find a kernel $k(\xi, t)$ of type $m$ which is a fundamental 
solution for $\widehat{L}$, that is,

$$
\widehat{L} k=\delta(\xi, t)
$$

in the sense of distributions, where $\delta$ is the delta distribution on $\mathfrak{g}^{\prime}$.

We denote by $U, U_{1}$ neighborhoods of the origin in $S(x)$, and by $V, V_{1}$ neighborhoods of the origin in $H(x)$ which are sufficiently small and satisfy $U \Subset U_{1}$, $V \Subset V_{1}$. Let $\varphi \in C_{0}^{\infty}\left(U_{1}\right), \varphi=1$ on $U, \psi \in C_{0}^{\infty}\left(V_{1}\right), \psi=1$ on $V$, and $\varrho(t) \in C_{0}^{\infty}(-2,2), \varrho(t)=1$ for $|t|<1$. We now define

$$
k_{0}(\xi, t)=\varphi \psi \varrho k(\xi, t) .
$$

From the definitions of the operators $\widehat{L}$ and $\widetilde{L}$ we see that

$$
\widetilde{L}=\widehat{L}+R,
$$

where $R$ has degree at most $m-1$. Consequently, for any $s \in \mathbb{N}$, the operator $\widetilde{L}$ can be written in the form

$$
\widetilde{L}=\widehat{L}+\sum_{i=1}^{s} R_{i}+Q_{s},
$$

where the $R_{i}$ are homogeneous operators of degree $m-i$ and $Q_{s}$ has degree at most $m-s-1$ at 0 . Using (5) and Lemma 1 we obtain

$$
\widetilde{L} k_{0}(\xi, t)=\varphi \psi \varrho \cdot \delta+\sum_{i=1}^{s} \varphi \psi \varrho k r_{i}+q_{s}
$$

for $\xi \in U_{1} \times V_{1}, t \in(-2,2)$, where $r_{i} \in H F_{i}, q_{s} \in F_{s+1}$.

Lemma 4. Given $s \in \mathbb{N}$ there exists a function $K_{s}(\xi, t) \in S F_{m}$ such that

$$
\widetilde{L} K_{s}=\varphi \psi \varrho \cdot \delta+H_{s}, \quad H_{s} \in S F_{s} .
$$

Proof. We use induction on $s$. For $s=0$ we set $K_{0}(\xi, t)=k_{0}(\xi, t)$, and the statement of the lemma follows from (6). Assume that it is true for $s-1$; then we have

$$
\widetilde{L} K_{s-1}=\varphi \psi \varrho \cdot \delta+H_{s-1}, \quad H_{s-1} \in S F_{s-1} .
$$

We now define $K_{s}(\xi, t)$ by $K_{s}=K_{s-1}-a(\xi, t) k_{0} * H_{s-1}$, where $a(\xi, t) \in C_{0}^{\infty}\left(\mathfrak{g}^{\prime}\right)$, supp $a \subset U_{1} \times V_{1} \times(-2,2)$ and $a \equiv 1$ in supp $H_{s-1}$. We have

$$
\widetilde{L} K_{s}=\varphi \psi \varrho \cdot \delta+H_{s-1}-a H_{s-1}+H_{s},
$$

where $H_{s}=a(\xi, t) \widehat{L} k_{0} * H_{s-1}-\widetilde{L}\left(a(\xi, t) k_{0} * H_{s-1}\right)$. By Lemma 1 it is clear that $K_{s}(\xi, t) \in S F_{m}, H_{s} \in S F_{s}$ and the proof is finished.

By Sobolev's embedding theorem for any $p \in \mathbb{N}$ there exists $s$ so that $S F_{\lambda} \subset$ $C^{p}(\mathfrak{g})$. From this fact and the previous lemma

$$
\widetilde{L} K_{s}=\varphi \psi \varrho \cdot \delta+H_{s}, \quad H_{s} \in C^{s}(\mathfrak{g}) .
$$

We now want to construct a fundamental solution for the original operator $L$. Set

$$
p_{s}(u, t)=\int K_{s}(u, v, t) d v, \quad h_{s}(u, t)=\int H_{s}(u, v, t) d v .
$$


Lemma 5. $L p_{s}=\varphi \varrho \cdot \delta+h_{s}$.

Proof. Let $R=L-\widetilde{L}$. Then

$$
L p_{s}=\varphi \varrho \cdot \delta+h_{s}-\int R K_{s} d v+\int R H_{s} d v .
$$

The operator $R$ is selfadjoint and acts only in the $v$ variables so it is easy to see that $\int R K_{s} d v=0$ and $\int R H_{s} d v=0$, and the lemma is proved.

Using the second property of the map $\Theta$ from Theorem 3 one can show that in the original coordinate system $(y)$ in some small neighborhood $\omega$ of the point $x \in M$,

$$
L v(x) p_{s}=\delta+h_{s}
$$

where $v(x)=\left|\operatorname{det}\left(\left.\mu_{x}\right|_{S(x)}\right)\right|$. This formula and Lemma 5 imply that

$$
U(x, x, t)=v(x) p_{s}(0, t)+g(t),
$$

where $g(t) \in C^{s}(\omega \times(-1,1))$. By construction,

$$
p_{s}(0, t)=\sum_{j=1}^{s} \int k_{j}(0, v, t) d v+\ldots
$$

If $k_{j} \in H F_{\lambda}$ for $\lambda<\beta(x)$ then by definition of this class

$$
\int k_{j}(0, v, t) d v=\int \widehat{k}_{j}(0, v, t) d v+\int g_{j}(0, v, t) d v .
$$

Consequently, $p_{j}(0, t)=\widehat{p}_{s}(0, t)+g(t)$, where $\widehat{p}_{s}(0, t)$ is homogeneous of degree $\lambda-\beta(x)$ and $g \in C^{\infty}(-1,1)$.

If $\lambda>\beta(x)$ then we have

$$
\partial_{t}^{a} p_{j}(0, t)=\int \partial_{t}^{a} k_{j}(0, v, t) d v+\int \partial_{t}^{a} c(0, v, t) d v=\widehat{p}_{j}(0, t)+g(t) .
$$

The function $\widehat{p}_{j}(0, t)$ is homogeneous of degree $(j-Q) / m-a$. If $(j-Q) / m-a \notin \mathbb{Z}$ then $\widehat{p}_{j}=c_{j} t^{(j-Q) / m-a}$, and after integrating over $t$ we obtain

$$
p_{j}(0, t)=c_{j} t^{(j-Q) / m}+g(t),
$$

$g \in C^{\infty}(-1,1)$. If $(j-Q) / m-a \in \mathbb{Z}$ then $\widehat{p}_{j}=c_{j} t^{-1}$ and so in this case

$$
p_{j}(0, t)=c_{j} t^{(j-Q) / m} \ln (t)+d_{j} t^{(j-Q) / m}+g(t),
$$

$g \in C^{\infty}(-1,1)$. From (7)-(9) it follows that for any $s \in \mathbb{N}$

$$
\left|U(x, x, t)-\sum_{j=-q(x)}^{s} c_{j}(x) t^{j / m}-\sum_{j=0}^{s} d_{j}(x) t^{j / m} \ln (t)\right|<C_{s} t^{(s+1) / m}
$$

and the proof of Theorem 1 is finished.

From the proof of Theorem 1 one can find the leading coefficient $c_{-q(x)}$ explicitly. It is clear that

$$
c_{-q(x)}=v(x) \cdot \int k(0, v, 1) d v,
$$


where $v(x)=\left|\operatorname{det}\left(\left.\mu_{x}\right|_{S(x)}\right)\right|$ and $k(u, v, t)$ is a fundamental solution for the operator $\widehat{L}$.

For the proof of Theorem 2 we observe that in the Métivier case $\Theta_{x}$ is smooth in $x \in M$ and so the asymptotic formula of Theorem 1 is uniform in $x$. Consequently, to obtain the statement of Theorem 2 we just integrate this formula over the manifold $M$.

\section{References}

[1] R. Beals and N. Stanton, The heat equation for the $\bar{\partial}$-Neumann problem I, Comm. Partial Differential Equations 12 (4) (1987), 407-413.

[2] G. Ben Arous, Noyau de la chaleur hypoelliptique et géométrie sous-riemannienne, in: Lecture Notes in Math. 1322, Springer, 1988, 1-16.

[3] -, Développement asymptotique du noyau de la chaleur hypoelliptique sur la diagonale, Ann. Inst. Fourier (Grenoble) 39 (1) (1989), 73-99.

[4] G. B. Folland, Subelliptic estimates and function spaces on nilpotent Lie groups, Ark Mat. 13 (1975), 161-207.

[5] B. Helffer et J. Nourrigat, Approximation d'un système de champs de vecteurs et applications à l'hypoellipticité, ibid. 17 (2) (1979), 237-254.

[6] —, - , Hypoellipticité maximale pour des opérateurs polynômes de champs de vecteurs, Progr. Math. 58, Birkhäuser, 1985.

[7] L. Hörmander, Hypoelliptic second order differential equations, Acta Math. 119 (1967), 147-171.

[8] D. Jerison and A. Sánchez-Calle, Estimates for the heat kernel for a sum of squares of vector fields, Indiana Univ. Math. J. 35 (4) (1986), 835-854.

[9] —, - Subelliptic second order differential operators, in: Lecture Notes in Math. 1287, Springer, 1988, 46-77.

[10] A. M. Lopatnikov, Asymptotic behaviour of the spectral function for operators constructed from vector fields, Vestnik Moskov. Univ. Ser. I Mat. Mekh. 1987 (3), 76-78 (Moscow Univ. Math. Bull. 42 (3) (1987), 70-72).

[11] - , Asymptotic behaviour of the spectral function for a class of hypoelliptic operators, Vestnik Moskov. Univ. Ser. I Mat. Mekh. 1988 (1), 92-94.

[12] - Spectral asymptotics for a class of hypoelliptic operators, manuscript, Moscow 1987, 94 pp., VINITI no. 2530 B87.

[13] G. Métivier, Fonction spéctrale et valeurs propres d'une classe d'opérateurs non elliptiques, Comm. Partial Differential Equations 1 (5) (1976), 467-519.

[14] O. Oleŭnik and E. Radkevich, Second Order Equations with Nonnegative Characteristic Form, Amer. Math. Soc., Providence, R.I., 1973.

[15] A. Sánchez-Calle, Fundamental solutions and geometry of the sum of squares of vector fields, Invent. Math. 78 (1984), 143-160.

[16] L. P. Rothschild, A criterion for hypoellipticity of operators constructed from vector fields, Comm. Partial Differential Equations 4 (1989), 645-699.

[17] L. P. Rothschild and E. M. Stein, Hypoelliptic differential operators and nilpotent Lie groups, Acta Math. 137 (3-4) (1976), 247-320.

[18] M. Taylor, Noncommutative microlocal analysis, Mem. Amer. Math. Soc. 313 (1984).

[19] T. Taylor, A parametrix for step two hypoelliptic diffusion equations, Trans. Amer. Math. Soc. 296 (1) (1986), 191-215.

[20] N. T. Varopoulos, Analysis on Lie groups, J. Funct. Anal. 76 (1988), 346-410. 